Hors-série | 2015

Les élites grecques modernes, $X V I^{\mathrm{e}}-\mathrm{XX}$ siècles :

identités, modes d'action, représentations

\title{
Les élites gouvernementales en Grèce, en Roumanie et en Espagne
}

Un regard comparatif sur leur composition actuelle

The Government Elites in Greece, Romania and Spain: a Comparative Approach

on their today Composition

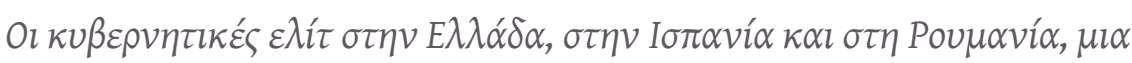

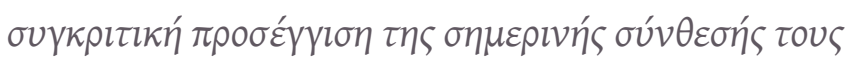

\section{Ekkehard W. Bornträger}

\section{Q OpenEdition}

Journals

Édition électronique

URL : https://journals.openedition.org/ceb/5747

DOI : $10.4000 / c e b .5747$

ISSN : 2261-4184

Éditeur

INALCO

Édition imprimée

Date de publication : 1 mars 2015

ISBN : 978-2-85837-224-5

ISSN : 0290-7402

Référence électronique

Ekkehard W. Bornträger, « Les élites gouvernementales en Grèce, en Roumanie et en Espagne ». Cahiers balkaniques [En ligne], Hors-série | 2015, mis en ligne le 26 janvier 2016, consulté le 06 juillet 2021. URL : http://journals.openedition.org/ceb/5747 ; DOI : https://doi.org/10.4000/ceb.5747

Ce document a été généré automatiquement le 6 juillet 2021.

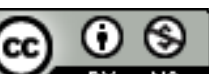

Cahiers balkaniques est mis à disposition selon les termes de la Licence Creative Commons Attribution - Pas d'Utilisation Commerciale 4.0 International. 


\title{
Les élites gouvernementales en Grèce, en Roumanie et en Espagne
}

\author{
Un regard comparatif sur leur composition actuelle \\ The Government Elites in Greece, Romania and Spain: a Comparative Approach \\ on their today Composition

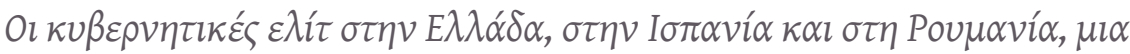

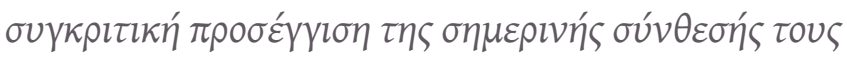

\section{Ekkehard W. Bornträger}

1 La crise actuelle en Grèce, omniprésente dans les médias locaux et internationaux, a également mis sous le feu des projecteurs l'élite politique du pays. Au-delà d'un large public, nombre de spécialistes en sciences économiques et sociales se sont attachés à découvrir certains aspects de ce groupe social et, surtout, ses réels ou prétendus dysfonctionnements. Rien de surprenant, donc, à ce que les tentatives d'explication théorique - pour ne rien dire des clichés populaires - qui circulent à son sujet soient légion, sans pour autant toujours faire justice à une réalité souvent complexe, voire pétrie de contradictions.

2 Sans prétendre rivaliser, pour la richesse de la documentation et l'analyse détaillée, avec les recherches de Koutsoukis (1982) et de Sotiropoulos et Bourikos (2012), notre objectif sera d'abord de compléter leur regard historique par une sorte d'instantané statistique des deux gouvernements grecs récents que sont ceux, respectivement, de Yorgos Papandréou et d'Antonios Samaras. Ce faisant, nous nous proposons d'élargir la perspective synchronique par une vision comparatiste en juxtaposant les paramètres grecs à ceux d'un autre pays de l'Europe du Sud-Est, la Roumanie, et à ceux d'un autre État méditerranéen, l'Espagne, tout en incluant la France comme référence «hors zone ». Cette perspective synchronique transnationale nous permettra de mieux faire ressortir les traits spécifiques - mais aussi les ressemblances - de l'élite ministérielle de chacun des pays examinés. Par ailleurs, elle nous fournira un point de départ pour quelques investigations rétrospectives comparées fondées sur la documentation existante. 


\section{Répartition géographique}

Ce sont les gouvernements grecs qui, dans notre échantillon, montrent le plus grand centralisme quant au recrutement géographique de leurs membres en fonction du lieu de naissance. La prédominance de l'agglomération athénienne est écrasante : $42 \%$, une proportion dépassant nettement celle du légendaire centralisme hexagonal. Ajoutons que les "villages" ( 10000 habitants) envoient très rarement un des leurs au gouvernement grec, tandis que dans les trois autres pays examinés presque un tiers des ministres ont des origines villageoises. L'exode rural en Grèce n'ayant pas été plus important que partout ailleurs, cet état de fait pourrait indiquer un circuit de renouvellement plus fermé, focalisé avant tout sur les élites urbaines établies, où la cooptation de nouveaux membres venus $\mathrm{du}$ « fond » de la province reste une exception. Cette hypothèse cadre bien, d'ailleurs, avec le constat qu'historiquement les régions de l'Attique, du Péloponnèse et de la $\Sigma \tau \varepsilon \dot{p} \varepsilon \alpha$ E $E \lambda \alpha \alpha ́ \delta \alpha$ ont été très longtemps

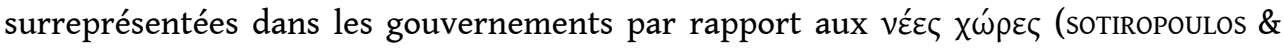
BOURIKOS, 2003, 174-178) entrées dans l'État grec en 1913 ou en 1947.

Quant à la composition géographique des cabinets espagnols et roumains, on est frappé par l'inversion de la proportion entre le centralisme du système politique et l'importance de la capitale comme "pépinière » de ministres. Si la Roumanie hypercentralisée puise à peine $15 \%$ de ses ministres dans l'agglomération de Bucarest, l'Espagne largement décentralisée, avec ses nombreuses régions autonomes, compte près de $30 \%$ de ministres madrilènes.

\section{Niveau d'études}

5 À en juger par le bagage scientifique et les honneurs académiques qui en sont l'illustration, les cabinets grecs et espagnols examinés ici rappelleraient presque l'idéetype de la République des savants selon Platon. Mais la Grèce excelle davantage, sur le plan des mérites académiques de ses dirigeants, que la monarchie ibérique. Ce sont ses ministres qui détiennent le record en matière de diplômes et autres doctorats obtenus auprès de hautes écoles étrangères - souvent les plus prestigieuses ${ }^{1}$. On discerne là un trait caractéristique des élites grecques, un phénomène qui s'est développé dès la fin du XIX ${ }^{e}$ siècle. Déjà, les Premiers ministres Trikoupis et Zaïmis avaient obtenu leurs titres académiques en France et en Allemagne. Le nombre d'inscrits auprès d'une université étrangère était encore modeste à l'époque, puisqu'il ne dépassait pas $10 \%$ des effectifs

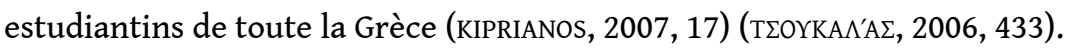

C'est ainsi qu'un observateur trop sensible aux lauriers académiques internationaux serait tenté de ne voir dans les derniers gouvernements de la France qu'une assemblée de provinciaux pauvres d'esprit - ou, en tout cas, pauvres en diplômes post-grades. La Roumanie, elle aussi, devance clairement la France quant au degré de "professoralisation» et d'« internationalisation» de ses ministres, sans pour autant égaler les deux pays méditerranéens. L'Hexagone pourrait trouver une consolation dans sa capacité d'afficher un " coefficient académique " plus élevé qu'une démocratie aussi prospère - et, à certains égards, modèle - que la Suède, dont les membres du 
gouvernement se contentent généralement du B.A., quand ils n'ont pas préféré mettre un terme à leurs études sans avoir décoché de diplôme.

7 Il serait certes aberrant de prétendre mesurer la qualité d'un gouvernement uniquement à la lumière des palmes académiques de ses membres, d'autant plus que l'exercice du pouvoir requiert souvent d'autres qualités que celles qui font un bon savant. Il n'en reste pas moins surprenant que la France, ainsi que les pays de l'Europe centrale et la Scandinavie, qui possèdent en général un savoir-faire technique et scientifique plus avancé que les sociétés méditerranéennes et balkaniques, semblent faire preuve d'une surprenante modestie en matière de profil universitaire de leurs gouvernants - sans toutefois que celle-ci ait des retombées négatives sur leurs performances. Mais peut-être faudrait-il inverser la question?

8 En effet, au cours des dernières années, plusieurs scandales relatifs aux antécédents académiques des membres du gouvernement ont secoué différents pays de l'Europe centrale et du Sud-Est. Deux ministres allemands ont ainsi perdu leur poste, dont curieusement - celle qui avait le portefeuille de l'Éducation et de la recherche scientifique. Le président hongrois démissionna en 2012 pour avoir plagié sa thèse de doctorat, tandis que le Premier ministre roumain, Victor Ponta, lui aussi convaincu de plagiat, conserva ses fonctions, sa ministre de l'Éducation faisant même pression ouvertement sur la commission académique de l'Université de Bucarest chargée de l'enquête.

9 Évidemment, il ne serait pas moins erroné de voir dans le grand nombre de ministres dotés de titres et/ou de fonctions académiques en Grèce et en Espagne, le seul fruit de pratiques académiques douteuses! Dans le cas grec, la fréquence des études dans les universités étrangères de haut niveau semble écarter le risque de manipulations «faciles» ou de protection politique. Des chercheurs éminents parvenus à un poste gouvernemental, tels Louka Katseli ou Évanghélos Liviératos, n'ont sans doute pas eu besoin de se faire épauler politiquement dans leur parcours universitaire. Il n'en demeure pas moins que les carrières académiques de certains futurs ministres dans leur pays d'origine semblent se dérouler avec une plus grande célérité que celles du commun des chercheurs. Quoi qu'il en soit, les interventions politiques plus ou moins discrètes dans les carrières universitaires sont fréquentes en Grèce.

10 Le "modèle méditerranéen" d'une plus grande participation du personnel universitaire au sommet du pouvoir exécutif et dans les hauts rangs des partis politiques nous apparaît davantage comme l'expression d'une intense politisation (et dépendance politique) du monde académique que comme l'indice d'une «imprégnation» scientifique notoire de l'action gouvernementale ou politique en général.

11 Ce manque de séparation entre les sphères politique et universitaire s'observe d'ailleurs dans certains régimes dictatoriaux ou autoritaires, dès lors qu'ils attribuent un grand prestige aux signes de distinction académique. C'est ainsi qu'ils excellent souvent par le haut niveau de qualification universitaire de leurs ministres. Près d'un tiers des membres du gouvernement espagnol de l'époque franquiste étaient titulaires d'un doctorat (GENEIEYS, 2004, 187). Au Portugal de l'Estado novo sous Salazar, la catégorie professionnelle la mieux représentée au gouvernement était celle des professeurs d'université (33\%), avant même les officiers militaires (26\%) (TAVRES DE ALMEIDA \& COSTA PINTO, 2003, 25-26). 
12 Considéré sous un autre angle, ce modèle de cooptation des cadres supérieurs universitaires trahit un recrutement assez exclusif, confortant l'hypothèse d'un système de circulation des élites politiques relativement fermé dans la péninsule ibérique et en Grèce - où le phénomène est particulièrement flagrant, les études à l'étranger étant en passe de devenir un présupposé pour quiconque veut faire partie des élites gouvernementales - et non un trait caractéristique de ceux qui, issus d'un milieu modeste, sont la première génération à accéder aux études supérieures.

\section{Les femmes au gouvernement}

13 Si le haut niveau de formation académique confère au système de recrutement ministériel grec - à première vue, certes, et abstraction faite de la problématique sousjacente - une position avantageuse face à la France, on constate l'inverse en matière de gender politics. La proportion des femmes dans les cabinets grecs reste plutôt modeste; un personnage aussi exceptionnel que Dora Bakoyanni mis à part, les quelques femmes qui occupent un ministère ne se voient guère attribuer de portefeuilles majeurs. À signaler, à cet égard, que même le ministère de la Défense, bastion masculin par excellence, est assuré ou a été assuré par des femmes en Espagne, en France, en Allemagne et en Suède, tandis qu'en Angleterre, en Allemagne, en Scandinavie et dans les pays baltes, des femmes dirigent ou ont dirigé le gouvernement. On a vu, au sein du gouvernement de Yorgos Papandréou, une tentative d'augmenter le nombre de portefeuilles féminins, mais, comme dans le cas de son homologue espagnol Zapatero qui avait même réussi l'exploit de porter le pourcentage des femmes au gouvernement à $50 \%$ - les rênes du pouvoir sont finalement restées en mains masculines. Dans le cas espagnol, on observa une prolifération de «mini-portefeuilles » qui, selon les dires de certains, servirent en priorité à rééquilibrer le gender ratio du cabinet. Il n'en est pas moins vrai que l'Espagne présente une image d'ensemble plus positive que la Grèce pour ce qui est de la présence des femmes au gouvernement.

Curieusement, tel n'est pas le cas d'un pays au passé socialiste comme la Roumanie, où les femmes jouissaient de jure des mêmes droits que les hommes et où leur intégration au marché du travail était devenue une réalité quotidienne depuis bien longtemps, même si leur nombre au sommet de la pyramide sociale était demeuré modeste. À l'époque où, en Espagne sous Franco, une femme mariée ne pouvait même pas ouvrir un compte en banque sans l'aval de son conjoint et où, en Grèce, la dot était encore inscrite dans la Constitution, la Roumanie nommait en 1947 en la personne d'Ana Pauker une femme à la tête du ministère des Affaires étrangères - ce qui lui valut à l'époque le titre de "femme la plus puissante d'aujourd'hui » dans le Time Magazine. En Grèce, il a fallu attendre près de 60 ans pour qu'une représentante de la gent féminine - qui n'était autre que Dora Bakoyanni - n'accède au même poste, en 2006. Cependant, on serait bien en peine aujourd'hui de trouver des traces de ce rôle de pionnier que la Roumanie avait joué dans la promotion des femmes à de hautes fonctions gouvernementales ${ }^{2}$. La Roumanie affiche la plus faible représentation féminine au gouvernement parmi les pays étudiés, avec à peine une ou deux membres du cabinet. Elle devance de peu l'Albanie, où le gouvernement Berisha comptait en 2013 une seule femme pour 16 ministres.

15 Il serait néanmoins prématuré de postuler un recul général du nombre des femmes au gouvernement dans les anciens pays socialistes. Les pays baltes donnent l'exemple 
contraire, et la Bulgarie a eu à partir de 1996 trois femmes au ministère des Affaires étrangères. Seule une étude détaillée pourrait nous apprendre s'il s'agit là de cas «illustres ", mais plutôt isolés, ou d'une tendance plus marquée.

Force est de constater que des pays comme la Grèce et, a fortiori, l'Espagne ont réussi un « rattrapage » impressionnant pour ce qui est de la présence des femmes aux postes suprêmes du pouvoir exécutif, quand bien même ils n'ont pas encore su combler entièrement le clivage qui, à cet égard, les sépare de la France, par exemple. Cette dernière est pourtant loin d'incarner le degré maximal de représentation égalitaire des sexes aux fonctions gouvernementales. Sur le plan quantitatif, en effet, les pays scandinaves se font forts d'une participation féminine encore plus élevée dépassant même la majorité, comme actuellement en Suède où le gouvernement Reinfeldt compte 8 femmes parmi ses 13 ministres. Et si la France n'a pas encore vu une femme diriger un gouvernement (sauf Édith Cresson du 15 mai 1991 au 2 avril 1992), même des pays moins avancés que la Scandinavie en termes d'émancipation féminine, telles la GrandeBretagne et l'Allemagne, ont déjà été dirigés par une femme - une dame de fer ou de plus souple constitution.

Les cabinets analysés laissent entrevoir une certaine coïncidence entre l'orientation politique du gouvernement et la présence de femmes en son sein. Surtout dans les cas grec, espagnol et roumain, un cabinet de gauche semble plus enclin à accepter des femmes dans ses rangs - alors qu'en France, l'alternance droite-gauche n'a qu'un faible impact sur le nombre des femmes ministres.

\section{Filières d'études et profil professionnel}

La professionnalisation croissante des élites ministérielles a pour conséquence que la durée d'exercice d'une profession autre que la politique a progressivement diminué, en Grèce et ailleurs. La militance politique commence souvent dès les études supérieures et, parfois, les spécialisations ultérieures comme la formation post-grade se font à un moment où la carrière politique même est déjà lancée, ce qui crée manifestement des interdépendances, voire des amalgames entre choix professionnels et choix politiques. De fait, à ce stade déjà, les candidats aspirant à une fonction politique se détachent de ceux qui s'orientent uniquement vers un avenir professionnel «normal», cette opposition s'accentuant encore après la fin des études. Pour ceux qui ambitionnent une place au pouvoir, la profession est souvent envisagée d'emblée comme une phase de transition visant à l'accumulation d'un capital « extra-politique », à seule fin de mieux se qualifier pour une carrière exclusivement politique.

19 Il en résulte que le profil professionnel des futures élites ministérielles s'avère généralement un peu flou - ou, du moins, mal délimité - échappant ainsi aux catégories traditionnelles. Pour cette raison, nous avons privilégié un classement sur la base de critères plus nets, à savoir les diplômes universitaires obtenus.

Ce qui saute aux yeux, dans les gouvernements grecs et espagnols, est la forte présence de « juristes » (dont les « administrateurs publics» tels que ceux qui, en France, sont issus de l'ENA) ${ }^{3}$. La Roumanie, en revanche, n'affiche qu'une faible participation de ce groupe professionnel, tandis que la France se situe quelque part au milieu, avec un clivage énorme entre les gouvernements Fillon et Ayrault, ce dernier se contentant de $16 \%$ de juristes seulement. Relativement faible est la part des spécialistes en sciences économiques et management, aussi bien en Grèce qu'en France. En revanche, la 
Roumanie et l'Espagne semblent offrir un terrain plus propice à l'exercice de leurs compétences. La Roumanie est d'ailleurs le seul pays de notre échantillon où les économistes arrivent en tête des groupes professionnels, une spécificité déjà apparente durant toute la phase de transition qui a suivi les bouleversements de 1989 (IONAșcu, 2007, 248-249).

21 Malgré la gloire et longévité de ses Lettres, la Grèce n'est point un lieu favorable à la carrière ministérielle des diplômés en lettres. Les chances de ces derniers sont les plus grandes dans la ville des Lumières, où rien moins que les deux derniers occupants du fauteuil de Premier ministre ont étudié les sciences humaines. En Grèce, le ministère de la Culture, animé un temps par l'artiste flamboyante qu'était Mélina Mercouri est aujourd'hui géré par des juristes ou des diplômés en sciences politiques. Quant à la patrie de Cervantès, elle aussi fait peu de cas des lettrés à ce niveau.

La terre promise du génie civil dans la carrière politique semble être la Roumanie où, selon nos échantillons, le quart des ministres sont issus de cette filière. Nous avons affaire ici à des spécialistes qui, à l'instar d'une partie des économistes, ont souvent fait une carrière à la fois technique et managériale dans l'agro-alimentaire ou les entreprises du secteur énergétique (idem, 249). Les cabinets grecs montrent aussi une préférence pour les diplômés en génie civil, tandis que la France, en dépit de son rôle pionnier dans l'élévation des «Ponts et chaussées" au rang d'orientation professionnelle fort respectable, leur ferme plutôt les portes du gouvernement.

Si l'on replace la composition professionnelle des gouvernements grecs dans leur contexte historique, la persistance d'une forte représentation de juristes est un signe de traditionalisme que la Grèce partage avec l'Espagne. En France, le quasi-monopole des avocats et des hauts magistrats au pouvoir exécutif a pris fin il y a près d'un demisiècle, d'abord avec l'arrivée des «cadres de l'administration publique » issus de l'ENA et d'autres grandes écoles (BIRNBAUM, 1977, 75-78), puis avec l'extension de la base de recrutement à d'autres catégories, à commencer par les sciences économiques et le management.

En Grèce, cette ouverture en est encore à ses prémices, tout comme en Espagne. En outre, dans ces deux pays plus longtemps qu'en France, un autre ensemble socioprofessionnel pratiquement disparu des cabinets aujourd'hui a su assurer sa représentation au gouvernement : celui des militaires qui, durant la première moitié du $\mathrm{xx}^{\mathrm{e}}$ siècle, était partout la pépinière habituelle de certains ministères. La persistance des dictatures en Espagne et au Portugal surtout, mais aussi, de manière plus passagère, le régime des colonels en Grèce, ont donné un sursis à la présence de hauts officiers au gouvernement, alors même qu'ils avaient déjà perdu tout rôle politique éminent dans les autres pays d'Europe occidentale - abstraction faite du général de Gaulle, bien sûr.

Nos échantillons ne révèlent pas de tendance généralisée à la préférence de catégories professionnelles spécifiques selon qu'il s'agit d'un gouvernement de droite ou de gauche. Cependant, en France - et, dans une moindre mesure, en Espagne - les cabinets de droite semblent accorder une certaine priorité aux juristes, la gauche privilégiant en revanche les économistes et les autres catégories professionnelles.

Dans l'ensemble, le profil socio-professionnel des élites ministérielles grecques laisse entrevoir beaucoup plus de similarités avec l'Espagne qu'avec la Roumanie, où les professions économiques et techniques dominent. En outre, de par son relatif conservatisme - forte dominance de juristes, niveau élevé de formation académique - il 
se distingue nettement de la situation observable aujourd'hui en France. Compte tenu de plusieurs autres particularités - grande importance des études à l'étranger, forte prévalence de l'agglomération athénienne comme base de recrutement - cette configuration dénote un système de renouvellement des élites assez exclusif, différent de la composition des gouvernements français récents, où l'on perçoit un notable élargissement de l'éventail socioprofessionnel et régional. Le verdict de кOUTSOUKIS (1982, 21), qui avait constaté il y a 30 ans un grand écart en Grèce entre la transformation très lente de l'élite et des changements socio-économiques nettement plus rapides semble valable aujourd'hui encore ${ }^{4}$.

Tandis que la Grèce et l'Espagne continuent se doter d'une élite politique triée sur le volet, la France, après avoir constitué, à l'instar de l'Angleterre, un cas typique de "social closure" (HARTMANN, 2007, 222), semble aujourd'hui évoluer vers un système de recrutement plus ouvert, se rapprochant du modèle de l'Europe centrale, de la Scandinavie et de l'Italie.

Certes, le très bon niveau académique des gouvernements grecs reste en soi impressionnant et quasiment inégalé, mais il semble n'avoir que peu d'impact concret et positif sur l'action gouvernementale. Au contraire, des figures politiques ayant à leur actif une impressionnante carrière scientifique internationale, tel l'ancien premier ministre Andréas Papandréou, se sont parfois montrées beaucoup moins à la hauteur des tâches qui leur incombaient qu'un certain homme d'État crétois, par exemple, qui avait juste fait des études de droit à Athènes, dont l'aéroport porte aujourd'hui son nom...

\section{BIBLIOGRAPHIE}

BIRNBAUM Pierre, 1977, les Sommets de l'État - Essai sur l'élite du pouvoir en France, Paris : Seuil. GENIEYS William, 2004, Las élites españolas ante el cambio de régimen político - Lógica de Estado y dinámicas centro-periferias en el siglo XX, Madrid: Centro de investigaciones sociológicas. HARTMANN Michael, 2007, Eliten und Macht in Europa - Ein internationaler Vergleich, Francfort-sur-leMain: Campus.

IONAŞCU Alexandra, 2007, «Perenitatea moştenirii comuniste?», in Raluca GROSESCU (dir.), Elitele comuniste înainte şi după 1989, Bucarest : Polirom, p. 231-264.

KYPRIANOS Pantelis, 2007, «La formation des élites grecques dans les universités occidentales ", Histoire de l'éducation, vol. 113, p. 3-30.

<http://histoire-education.revues.org/1354> KOUTSOUKIS Kleomenis, 1982, Political Leadership in Modern Greece: Cabinet Elite Circulation and Systemic Change, Athènes: Athena.

SOTIROPOULOS Dimitris \& BOURIKos Dimitris, 2012, “Ministerial Elites in Greece, 1843-2001: A Synthesis of old Sources and New Data", in Pedro TAVARES DE ALMEIDA, Antonio COSTA PINTO \& 
Nancy BERMEO (dir.), Who governs Southern Europe - Regime Change and Ministerial Recruitment 1850-2000, New York: Routledge, pp. 153-204.

TAVARES DE ALMEIDA Pedro \& Antonio costa PINTO, (2012), “Portuguese Ministers, 1851-1999: Social Background and Paths to Power", in Pedro tAVARES DE ALMEIDA, Antonio costA PINTO \& Nancy BERMEO (dir.)., Who governs Southern Europe - Regime Change and Ministerial Recruitment 1850-2000, New York: Routledge, pp. 5-40.

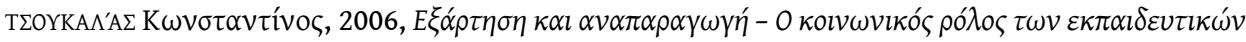

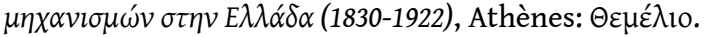

\section{ANNEXES}

\section{Quelques données statistiques récentes sur les cabinets grec, roumain, espagnol et français}

\begin{tabular}{|c|c|c|c|c|}
\hline & Grèce & Roumanie & Espagne & France \\
\hline & Cabinet & Cabinet & Cabinet & Cabinet \\
\hline & A & $\begin{array}{l}\text { A } \\
\text { Boc } 1\end{array}$ & $\begin{array}{l}\text { A } \\
\text { Zapatero }\end{array}$ & $\begin{array}{l}\text { A } \\
\text { Fillon } 1\end{array}$ \\
\hline & $\begin{array}{l}\text { B } \\
\Sigma \alpha \mu \alpha \rho \alpha ́ \alpha\end{array}$ & $\begin{array}{l}\text { B } \\
\text { Ponta } 2\end{array}$ & $\begin{array}{l}\text { B } \\
\text { Rajoy }\end{array}$ & $\begin{array}{l}\text { B } \\
\text { Ayrault } 1\end{array}$ \\
\hline & $C=(A+B) / 2$ & $\mathrm{C}=(\mathrm{A}+\mathrm{B}) / 2$ & $C=(A+B) / 2$ & $C=(A+B) / 2$ \\
\hline Date & $\begin{array}{l}\text { A } 6-10-2009 \\
\text { B 21-6-2012 }\end{array}$ & $\begin{array}{l}\text { A } 23-12-2009 \\
\text { B déc. } 2012 \\
\text { au } 23-6-13^{1}\end{array}$ & $\begin{array}{l}\text { A 12-4-2008 } \\
\text { B déc. } 2011 \\
\text { au 23-6-13 }\end{array}$ & $\begin{array}{l}\text { A } 18-5-2007 \\
\text { B } 17-5-2012\end{array}$ \\
\hline $\begin{array}{l}\text { Nombre } \\
\text { des } \\
\text { ministres }\end{array}$ & $\begin{array}{l}\text { A } 16 \\
\text { B } 18 \\
\text { C total } 34\end{array}$ & $\begin{array}{ll}\text { A } & 18 \\
\text { B } & 19 \\
\text { C } & \text { total } 37\end{array}$ & $\begin{array}{ll}\text { A } & 21 \\
\text { B } & 14 \\
\text { C } & \text { total } 35\end{array}$ & $\begin{array}{ll}\text { A } & 16 \\
\text { B } & 19 \\
\text { C } & \text { total } 35\end{array}$ \\
\hline \multicolumn{5}{|c|}{ Y compris le Premier ministre } \\
\hline $\begin{array}{l}\text { Données } \\
\text { suivantes } \\
\text { en \% }\end{array}$ & GR & RO & ES & FR \\
\hline $\begin{array}{l}\text { Part des } \\
\text { femmes }\end{array}$ & $\begin{array}{lr}\text { A } & 31,3 \\
\text { B } & 5,6 \\
\text { C } & 17,6\end{array}$ & $\begin{array}{rr}\text { A } & 6 \\
\text { B } & 11 \\
\text { C } & 8\end{array}$ & $\begin{array}{ll}\text { A } & 43 \\
\text { B } & 29 \\
\text { C } & 37\end{array}$ & $\begin{array}{l}\text { A } 44 \\
\text { B } 47 \\
\text { C } 46\end{array}$ \\
\hline $\begin{array}{l}\text { Part des } \\
\text { pers. nées } \\
\text { à l'étranger }\end{array}$ & $\begin{array}{lr}\text { A } & 12,5 \\
\text { B } & 5,6 \\
\text { C } & 8,8\end{array}$ & - & - & $\begin{array}{lr}\text { A } & 6 \\
\text { B } & 21 \\
\text { C } & 14\end{array}$ \\
\hline
\end{tabular}




\begin{tabular}{|c|c|c|c|c|}
\hline $\begin{array}{l}\text { Âge moyen } \\
\text { (à la } \\
\text { formation } \\
\text { cabinet) }\end{array}$ & $\begin{array}{lc}\text { A } & 5 \\
\text { B } & 56 \\
\text { C } & 53,6\end{array}$ & $\begin{array}{ll}\text { A } & 49 \\
\text { B } & 40 \\
\text { C } & 44,3\end{array}$ & $\begin{array}{ll}\text { A } & 52,5 \\
\text { B } & 56,5 \\
\text { C } & 54,1\end{array}$ & $\begin{array}{ll}\text { A } & 54 \\
\text { B } & 54 \\
\text { C } & 53,8\end{array}$ \\
\hline $\begin{array}{l}\text { Lieu } \\
\text { Origine }\end{array}$ & GR & RO & ES & FR \\
\hline a) capitale & $\begin{array}{ll}\text { A } & 44 \\
\text { B } & 33 \\
\text { C } & 38\end{array}$ & $\begin{array}{ll}\text { A } & 11 \\
\text { B } & 11 \\
\text { C } & 11\end{array}$ & $\begin{array}{ll}\text { A } & 24 \\
\text { B } & 36 \\
\text { C } & 29\end{array}$ & $\begin{array}{ll}\text { A } & 13 \\
\text { B } & 16 \\
\text { C } & 14\end{array}$ \\
\hline $\begin{array}{l}\text { b) environ } \\
\text { capitale }\end{array}$ & $\begin{array}{ll}A & 6 \\
B & 0 \\
C & 3\end{array}$ & $\begin{array}{ll}\text { A } & - \\
\text { B } & 11 \\
\text { C } & 5\end{array}$ & - & $\begin{array}{ll}\text { A } & 19 \\
\text { B } 16 \\
\text { C } 17\end{array}$ \\
\hline $\begin{array}{l}\text { c) ville sauf } \\
\text { capitale } \\
\text { > } 100000\end{array}$ & $\begin{array}{ll}\text { A } & 19 \\
\text { B } & 17 \\
\text { C } & 18\end{array}$ & $\begin{array}{ll}\text { A } & 39 \\
\text { B } & 21 \\
\text { C } & 30\end{array}$ & $\begin{array}{l}\text { A } 33 \\
\text { B } 36 \\
\text { C } 34\end{array}$ & $\begin{array}{ll}\text { A } & 19 \\
\text { B } & 16 \\
\text { C } & 17\end{array}$ \\
\hline $\begin{array}{l}\text { d) village } \\
<10000\end{array}$ & $\begin{array}{ll}\text { A } & 13 \\
\text { B } & 17 \\
\text { C } & 15\end{array}$ & $\begin{array}{ll}\text { A } & 33 \\
\text { B } & 32 \\
\text { C } & 32\end{array}$ & $\begin{array}{lr}\text { A } & 19 \\
\text { B } & 7 \\
\text { C } & 14\end{array}$ & $\begin{array}{ll}\text { A } & 19 \\
\text { B } & 37 \\
\text { C } & 29\end{array}$ \\
\hline Niveau formation & GR & RO & ES & FR \\
\hline $\begin{array}{l}\text { a) } \\
\text { universitaire }\end{array}$ & $\begin{array}{l}\text { A } 100 \\
\text { B } 100 \\
\text { C } 100\end{array}$ & $\begin{array}{lr}\text { A } & 100 \\
\text { B } & 95 \\
\text { C } & 97\end{array}$ & $\begin{array}{lr}\text { A } & 95 \\
\text { B } & 100 \\
\text { C } & 97\end{array}$ & $\begin{array}{l}\text { A } 100 \\
\text { (ENA 25) } \\
\text { B } 100 \\
\text { (ENA 16) } \\
\text { C } 100 \\
\text { (ENA 20) }\end{array}$ \\
\hline $\begin{array}{l}\text { b) avec } \\
\text { doctorat }\end{array}$ & $\begin{array}{ll}\text { A } & 44 \\
\text { B } & 33 \\
\text { C } & 38\end{array}$ & $\begin{array}{ll}\text { A } & 22 \\
\text { B } & 37 \\
\text { C } & 30\end{array}$ & $\begin{array}{ll}\text { A } & 43 \\
\text { B } & 29 \\
\text { C } 37\end{array}$ & $\begin{array}{lr}\text { A } & 31 \\
\text { B } & 5 \\
\text { C } & 17\end{array}$ \\
\hline $\begin{array}{l}\text { c) études à } \\
\text { l'étranger } \\
\text { (> } 1 \text { an) }\end{array}$ & $\begin{array}{ll}\text { A } & 31-44 \\
\text { B } & 33-39 \\
\text { C } & 32-41\end{array}$ & $\begin{array}{rr}\text { A } & 17 \\
\text { B } & 6 \\
\text { C } & 11\end{array}$ & $\begin{array}{lr}\text { A } & 24 \\
\text { B } & 7 \\
\text { C } & 17\end{array}$ & $\begin{array}{ll}\mathrm{A} & 0 \\
\mathrm{~B} & 0 \\
\mathrm{C} & 0\end{array}$ \\
\hline $\begin{array}{l}\text { Filière } \\
\text { d'étude } \\
\text { (hors } \\
\text { politique) }\end{array}$ & GR & RO & ES & FR \\
\hline $\begin{array}{l}\text { a) droit, } \\
\text { administration } \\
\text { publique }\end{array}$ & $\begin{array}{ll}\text { A } & 44 \\
\text { B } & 44 \\
\text { C } & 44\end{array}$ & $\begin{array}{ll}\text { A } & 22 \\
\text { B } & 26 \\
\text { C } & 24\end{array}$ & $\begin{array}{ll}\text { A } & 43 \\
\text { B } & 50 \\
\text { C } & 46\end{array}$ & $\begin{array}{ll}\text { A } & 63 \\
\text { B } & 16 \\
\text { C } & 37\end{array}$ \\
\hline $\begin{array}{l}\text { b) sciences } \\
\text { économiques, } \\
\text { management }\end{array}$ & $\begin{array}{ll}\text { A } & 19 \\
\text { B } & 22 \\
\text { C } & 21\end{array}$ & $\begin{array}{ll}\text { A } & 28 \\
\text { B } & 37 \\
\text { C } & 32\end{array}$ & $\begin{array}{ll}\text { A } & 33 \\
\text { B } & 29 \\
\text { C } & 31\end{array}$ & $\begin{array}{ll}\text { A } & 13 \\
\text { B } & 32 \\
\text { C } & 23\end{array}$ \\
\hline c) lettres & $\begin{array}{ll}\text { A } & 0 \\
\text { B } & 6 \\
\text { C } & 3\end{array}$ & $\begin{array}{rr}\text { A } & 17 \\
\text { B } & 6 \\
\text { C } & 11\end{array}$ & $\begin{array}{cc}\text { A } & 10 \\
\text { B } & 0 \\
\text { C } & 6\end{array}$ & $\begin{array}{ll}\text { A } & 19 \\
\text { B } & 32 \\
\text { C } & 26\end{array}$ \\
\hline
\end{tabular}




\begin{tabular}{|c|c|c|c|c|}
\hline $\begin{array}{l}\text { d) } \\
\text { ingénierie }\end{array}$ & $\begin{array}{lr}\text { A } & 6 \\
\text { B } & 17 \\
\text { C } & 12\end{array}$ & $\begin{array}{ll}\text { A } & 28 \\
\text { B } & 21 \\
\text { C } & 24\end{array}$ & $\begin{array}{ll}\text { A } & 5 \\
\text { B } & 7 \\
\text { C } & 6\end{array}$ & $\begin{array}{ll}\text { A } & 0 \\
\text { B } & 5 \\
\text { C } & 3\end{array}$ \\
\hline $\begin{array}{l}\text { e) professeurs } \\
\text { des } \\
\text { universités }\end{array}$ & $\begin{array}{ll}\text { A } & 25 \\
\text { B } & 22 \\
\text { C } & 24\end{array}$ & $\begin{array}{rr}\text { A } & 6 \\
\text { B } & 11 \\
\text { C } & 8\end{array}$ & $\begin{array}{ll}\text { A } & 29 \\
\text { B } & 21 \\
\text { C } & 26\end{array}$ & $\begin{array}{ll}\text { A } & 6 \\
\text { B } & 5 \\
\text { C } & 6\end{array}$ \\
\hline
\end{tabular}

\section{Tableau diachronique de la filière universitaire ou post-secondaire des ministres en Grèce en comparaison avec des données historiques concernant l'Espagne, la Roumanie et la France. Données en pourcentages.}

\begin{tabular}{|c|c|c|c|c|c|c|}
\hline Grèce GR & 1910-1936 & 1936-1941 & 1946-1967 & $1967-1974$ & 1974-2001 & $\begin{array}{l}2009 / 2012 \\
\Pi \alpha \pi . / \Sigma \alpha \mu .\end{array}$ \\
\hline Espagne ES & 1931-1939 & & 1939-1974 & & & \\
\hline France FR & & & 1944-1969 & & & \\
\hline Roumanie RO & & & & & 1989-2004 & \\
\hline $\begin{array}{l}\text { Droit et } \\
\text { administration } \\
\text { publique }\end{array}$ & $\begin{array}{l}\text { GR } 54 \\
\text { ES } 57\end{array}$ & GR 30 & $\begin{array}{ll}\text { GR } & 65 \\
\text { ES } & 34 \\
\text { FR } & 36\end{array}$ & GR 41 & $\begin{array}{l}\text { GR } 45 \\
\text { RO } 12\end{array}$ & GR 44 \\
\hline $\begin{array}{l}\text { Économie et } \\
\text { management }\end{array}$ & & & $\begin{array}{lr}\text { GR } & 6 \\
\text { ES } & 4 \\
\text { FR } & 15\end{array}$ & GR 12 & $\begin{array}{l}\text { GR } 24 \\
\text { RO } 26\end{array}$ & GR 21 \\
\hline $\begin{array}{l}\text { Formation } \\
\text { militaire }\end{array}$ & GR 17ES 5 & GR 21 & $\begin{array}{ll}\text { GR } & 14 \\
\text { ES } & 33\end{array}$ & GR 25 & $\begin{array}{ll}\text { GR } & 3 \\
\text { RO } & 5\end{array}$ & - \\
\hline Ingénierie & $\begin{array}{ll}\text { GR } & 6,3 \\
\text { ES } & 4\end{array}$ & GR $\quad 2,9$ & $\begin{array}{ll}\text { FR } \quad 5,4 \\
\text { ES } 10\end{array}$ & GR 3 & $\begin{array}{l}\text { GR } 13 \\
\text { RO } 12\end{array}$ & GR 12 \\
\hline $\begin{array}{l}\text { Sciences } \\
\text { humaines }\end{array}$ & & & & GR 2 & GR 2 & GR 2 \\
\hline
\end{tabular}

\section{NOTES}

1. Cette propension massive aux études à l'étranger (environ $40 \%$ des ministres) semble être à l'évidence un signe distinctif du sommet de la classe politique grecque. Un contrôle des données pour d'autres pays européens de la même importance démographique (Portugal, Suède) montre qu'il ne s'agit pas d'une spécificité des "petits" pays, même si ceux-ci ont tendance à accueillir dans les rangs du gouvernement légèrement plus de diplômés des universités étrangères qu'une " grande » nation comme la France. Supposer un effet de diaspora n'aide pas non plus à 
expliquer ce phénomène, le gouvernement israélien ne comptant pas non plus un grand nombre de diplômés à l'étranger.

2. Il est vrai que ce procédé devait parfois prendre des formes caricaturales, au temps de la dictature familiale de Ceauşescu, où la femme du conducător menait la plus brillante carrière scientifique, se voyant projeter sans effort intellectuel particulier du rang de simple laborantine ayant à son actif à peine quelques années de scolarité d'ailleurs peu glorieuses - jusqu'au sommet de la recherche en chimie en Roumanie. Mais de telles aberrations dues au culte de la personne n'enlèvent rien au fait que le niveau réel de formation féminine - et de participation féminine au pouvoir - était en moyenne plus élevé qu'en Grèce.

3. Il est intéressant de voir qu'une "science du pouvoir » comme le droit a constitué, en Grèce et en Espagne, une formation fréquemment choisie (mais pas toujours menée à bien) dans le monde des lettres également. Qu'il suffise de mentionner des écrivains grecs tels Karyotakis, Élytis, Palamas, Petsalis-Diomidis, Cosmas Politis, Séféris, Théotokas, ou espagnols comme Clarín, Perez-Galdós, Valle-Inclán. Une analyse comparative approfondie permettrait de vérifier si, à cet égard aussi, se profile une spécificité commune aux deux pays.

4. Il est évident qu'une recomposition personnelle de l'élite gouvernementale, même dans le sens d'une "démocratisation " de l'accès à de telles fonctions, ne changera à elle seule pas grand-chose à l'art de gouverner en Grèce. En Italie, l'aboutissement décevant du mouvement des "mani pulite » et les dérapages d'un antiélitisme populiste (au niveau culturel, du moins) aux teintes poujadistes et/ou régionalistes l'illustrent de manière éclatante. Sans un changement de la mentalité politique et des structures qui la sous-tendent (clientélisme, corporatisme, institutions faibles, etc.) en faveur d'un système plus méritocratique, une modernisation des élites n'entraînera pas celle de la société.

1. Y compris le ministère des Affaires européennes créé le 20 septembre 2011.

\section{RÉSUMÉS}

Notre essai se propose de porter un regard "statistique » et comparatif sur la composition actuelle des élites ministérielles en Grèce, en Roumanie et en Espagne. Nous utiliserons des paramètres tels que la provenance géographique, le parcours académique, le profil professionnel et la gender distribution.

Our paper addresses some aspects of the present composition of ministerial elites in Greece, Romania and Spain by means of a comparative statistical approach comprising data on geographical origin, academic and professional background and gender distribution.

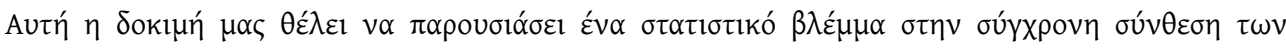

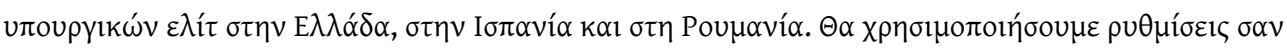

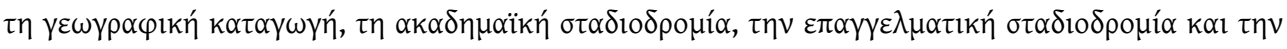

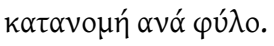


INDEX

Index géographique : Grèce, Espagne, Roumanie

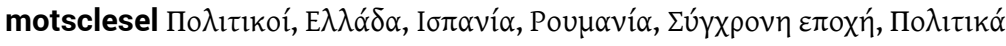

motsclestr Politikacılar, Yunanistan, İspanya, Romanya, Çağdaş, Siyaset

motsclesmk ПОЛИТИЧАРИ, ГРЦИЈА, ШПАНИЈА, РОМАНИЈА, СОВРЕМЕНА, ПОЛИТИКА

Thèmes : Politique

Keywords : Politicians, Greece, Spain, Rumania, Contemporary, Politics

Mots-clés : Personnel politique

Index chronologique : vingtième siècle -- fin, vingt-et-unième siècle -- début

\section{AUTEUR}

\section{EKKEHARD W. BORNTRÄGER}

Universités de Genève et de Fribourg 